\title{
Diophantinized Fractional Representations for Nonlinear Elastomeric Media
}

Joe Padovan

University of Akron

Jerzy T. Sawicki

Cleveland State University, j.sawicki@csuohio.edu

Follow this and additional works at: https://engagedscholarship.csuohio.edu/enme_facpub

Part of the Mechanical Engineering Commons

How does access to this work benefit you? Let us know!

Publisher's Statement

NOTICE: this is the author's version of a work that was accepted for publication in Computers \&

Structures. Changes resulting from the publishing process, such as peer review, editing,

corrections, structural formatting, and other quality control mechanisms may not be reflected in this document. Changes may have been made to this work since it was submitted for publication. A definitive version was subsequently published in Computers \& Structures, 66, 5, (03-01-1998); 10.1016/S0045-7949(97)00067-9

\section{Original Citation}

Padovan, J., and Sawicki, J.T. (1998) Diophantinized Fractional Representations for Nonlinear Elastomeric Media. Computers \& Structures, 66(2), 613-626, doi: 10.1016/S0045-7949(97)00067-9.

This Article is brought to you for free and open access by the Mechanical Engineering Department at EngagedScholarship@CSU. It has been accepted for inclusion in Mechanical Engineering Faculty Publications by an authorized administrator of EngagedScholarship@CSU. For more information, please contact library.es@csuohio.edu. 


\title{
DIOPHANTINIZED FRACTIONAL REPRESENTATIONS FOR NONLINEAR ELASTOMERIC MEDIA
}

\author{
Joe Padovan $\dagger$ and Jerzy T. Sawicki $\ddagger$ \\ $\uparrow$ Departments of Mechanical and Polymer Engineering. The University of Akron, Akron, \\ OH 44325-3903, U.S.A. \\ ¥Department of Mechanical Engineering, Cleveland State University, Cleveland, OH 44115, U.S.A.
}

\section{INTRODUCTION}

The analysis of mechanical components composed of elastomers poses several difficulties to the designer-analyst, namely: (i) large deformation and strain kinematics; (ii) complex boundary conditions; and (iii) nonlinear material properties. Because of the coiling/twisting and kinking/folding actions of their cross linked long chain molecules, polymers possess very different compressive and tensile properties [1]. Such behavior, if not properly represented, invariably leads to the anomalous performance of typical nonlinear finite element solution algorithms. This is usually evidenced by a lack of convergence, as well as various element related difficulties.

There are several constitutive modeling schemes for elastomers, namely by Mooney-Rivlin [2,3], Peng [4], Peng and Landel [5] and Ogden [6]. The work of Mooney [2], later modified by Rivlin [3], involves the use of invariants to define the strain energy. Through the first law, the three-dimensional stress-strain relation can then be extracted. By employing higher order polynomials in terms of the invariants, fairly complex responses can be handled. As an alternative, Peng [4] and Peng-Landel [5] caste the properties in terms of transcendental functions. More recently, Ogden [6] employed fractional representations in terms of the stretches. Noting the very useful paper of Finney and Kumar [7], the least square procedures can be employed to optimize the accuracy of the noted schemes. Of the procedures, perhaps the most accurate is the Ogden [6] approach. It provides the best fit for the least num- ber of terms. In higher order applications it becomes somewhat awkward to determine fitting coefficients, as well as polynomial powers.

In general, the problem of establishing constitutive models is one of defining the most appropriate basis space, i.e. one that provides the requisite completeness while maintaining physically admissible base functions. For instance, in the higher order Mooney-Rivlin model, the base functions are polynomials in the invariants, thus to improve the fit one must raise the order. Since the actual response does not possess such polynomial powers, higher order Mooney-Rivlin fits tend to become stiffer and thus less stable numerically.

To bypass such problems, this paper will explore the use of diophantinized fractional representations. These will be taken either in terms of the stretches or strain invariants. Both polynomial and rational forms will be considered in the development. To provide for the requisite base completeness and physical admissibility, the diophantinized set of fractional powers will be bound by the curvature properties of the empirical data set. Next, to obtain the most optimal fit within the required tolerance, a remezed [8-10] least square fitting procedure will be developed. This will include an iterative densification of the set of diophantinized fractional powers. Note, the densification will be achieved while maintaining physically admissible curvature bounds on the set.

In the sections which follow, detailed discussions will be given on: (i) a review of available fitting schemes along with their limitations; (ii) the first 
law [11-13] and its use in formulating the fit; (iii) the fractionalized polynomial fitting scheme along with its algorithmic implementation; (iv) the fractionalized rational fit; and (v) sample applications.

\section{FIRST LAW OF THERMO}

To provide a framework for the empirical curve fitting process, the first law of thermo is introduced. This requires the discussion of three main issues, i.e.:

1. what kinematic-stress measures need to be employed;

2. how to frame the work-energy conservation principle [11-13];

3. how to functionally characterize the work-energy term.

From an Euler point of view [12], the kinematicstress pair involves the stretch ratio $e_{i}$ and the Cauchy true stress $\sigma_{i}$. Here, $e_{i}$ is defined by the expression [11]

$$
e_{i}=\frac{L_{i}(t+\mathrm{d} t)}{L_{i}(t)}
$$

where $L_{i}$ are the rectilinear length measures. Given that $W$ represents the work-energy in principal stress-strain coordinates, the first law takes the form [11]

$$
\frac{\mathrm{d}}{\mathrm{d} t}(W)=\sigma_{i} \frac{\mathrm{d}}{\mathrm{d} t}\left(e_{i}\right)
$$

Since ultimately

$$
W=W\left(e_{1}, e_{2}, e_{3}\right),
$$

hence

$$
\frac{\mathrm{d}}{\mathrm{d} t}(W)=\frac{\partial}{\partial e_{i}}(W) \frac{\mathrm{d}}{\mathrm{d} t}\left(e_{i}\right)
$$

Based on Equation (4), Equation (2) yields the expression

$$
\left(\sigma_{i}-\frac{\partial}{\partial e_{i}}(W)\right) \frac{\mathrm{d}}{\mathrm{d} t}\left(e_{i}\right)=0 .
$$

For compressible media, since $e_{i}$ are linearly independent, it follows that Equation (5) leads to

$$
\sigma_{i}=\frac{\partial}{\partial e_{i}}(W)
$$

In the case of incompressible media, $e_{i}$ are linearly dependent. This follows from the fact that

$$
e_{1} e_{2} e_{3}=\frac{\mathrm{d} V_{t+\mathrm{d} t}}{\mathrm{~d} V_{t}},
$$

where $\mathrm{d} V$ defines the volume. For incompressible media

$$
e_{1} e_{2} e_{3} \equiv 1
$$

$$
e_{i} \sim 1+\mathrm{d} e_{i},
$$

eqn (2.8) yields that

$$
\begin{aligned}
e_{1} e_{2} e_{3}= & \left(1+\mathrm{d} e_{1}\right)\left(1+\mathrm{d} e_{2}\right)\left(1+\mathrm{d} e_{3}\right) \\
= & 1+\mathrm{d} e_{1}+\mathrm{d} e_{2}+\mathrm{d} e_{3}+\mathrm{d} e_{1} \mathrm{~d} e_{2}+\mathrm{d} e_{2} \mathrm{~d} e_{3} \\
& +\mathrm{d} e_{1} \mathrm{~d} e_{3}+\mathrm{d} e_{1} \mathrm{~d} e_{2} \mathrm{~d} e_{3} .
\end{aligned}
$$

For $\mathrm{d} e_{i} \rightarrow 0^{+}$, namely virtual stretching, eqn (10) reduces to the form

$$
\mathrm{d} e_{1}+\mathrm{d} e_{2}+\mathrm{d} e_{3} \sim 0 .
$$

Hence, $\mathrm{d} e_{i}$ are in the limit linearly dependent. When a purely hydrostatic situation arises, $\partial /$ $\partial e_{i}(W) \equiv 0$. In this context, $\sigma_{i}=-P$, i.e. the hydrostatic pressure. Now, in terms of Equation (5), either the terms are perpendicular or null. In the context of the hydrostatic case, it is unlikely that $\sigma i-\left(\partial / \partial e_{i}\right)(W)$ is zero. Rather, it is likely to be equal to pressure related parameters. These must all be the same to allow the use of Equation (11). Hence, we yield that

$$
\sigma_{i}=\frac{\partial}{\partial e_{i}}(W)-P .
$$

In a Lagrange framework [12], $e_{i}$ is replaced by the stretch ratio[11]

$$
\lambda_{i}=\frac{L_{t+\mathrm{d} t}}{L_{0}} .
$$

Based on Equation (13), it follows that

$$
\mathrm{d} e_{i}=\mathrm{d} \lambda_{i} / \lambda_{i}
$$

Similarly, $\sigma_{i}$ and $P$ must be replaced by their second Piola-Kirchhoff equivalents. The conservation of mass principle requires that

$$
\rho_{0} \mathrm{~d} V_{0}=\rho_{t+\mathrm{d} t} \mathrm{~d} V_{t+\mathrm{d} t},
$$

where $\rho$ are the densities.

Since

$$
\lambda_{1} \lambda_{2} \lambda_{3}=\frac{\mathrm{d} V_{t+\mathrm{d} t}}{\mathrm{~d} V_{0}}
$$

we get the expression

$$
\lambda_{1} \lambda_{2} \lambda_{3}=\rho_{0} / \rho_{t+\mathrm{d} t} .
$$

In the principal orientations the Cauchy [13] and second Piola-Kirchhoff [13] stresses are related by the relationship

$$
\sigma_{i}\left(L_{l} L_{k}\right)_{t+\mathrm{d} t}=S_{i}\left(L_{i} L_{k}\right)_{0},
$$

where $i \neq l \neq k$. Given Equation (17) we see that after several algebraic manipulations

$$
\sigma_{i}=\frac{\rho_{t+\mathrm{d} t}}{\rho_{0}} \lambda_{i} S_{i}
$$

Given that 


$$
p=\frac{\rho_{t+\mathrm{d} t}}{\rho_{0}} \lambda_{i} P
$$

Employing Equation (14) and Equations (19) and (20), Equation (6) reduces the Lagrangian from given by the relation

$$
{ }_{i}=\frac{\rho_{0}}{\lambda_{t+\mathrm{d} t}} \frac{\partial}{\partial \lambda_{i}}(W)
$$

For the incompressible case Equation (12), we yield

$$
{ }_{i}=\frac{\partial}{\partial \lambda_{i}}(W)-P
$$

Next we must explore the functional form of $W$. Mooney [2] in his early paper caste the work-energy in terms of the strain invariants, i.e.

$$
\begin{gathered}
I_{1}=\lambda_{1}^{2}+\lambda_{2}^{2}+\lambda_{3}^{2} \\
I_{2}+\left(\lambda_{1} \lambda_{2}\right)^{2}+\left(\lambda_{1} \lambda_{3}\right)^{2}+\left(\lambda_{2} \lambda_{3}\right)^{2} \\
I_{3}=\lambda_{1} \lambda_{2} \lambda_{3}
\end{gathered}
$$

In this context, following Mooney [2] and Rivlin [3]

$$
W=W\left(I_{1}-3, I_{2}-3, I_{3}-1\right)
$$

For incompressible media, since $I_{3} \rightarrow O(1)$, Equation (26) reduces to

$$
W=W\left(I_{1}-3, I_{2}-3\right)
$$

From a functional point of view, either $W$ is a general form wherein the $I_{1}, I_{2}, I_{3}$ terms are coupled, or it is additively decomposable, i.e.

$$
W=W_{1}\left(I_{1}-3\right)+W_{2}\left(I_{2}-3\right)+W_{3}\left(I_{3}-1\right)
$$

This can be sorted out during the empirical curve fitting process.

Alternatively, following Peng [4], Peng-Landel [5] and Ogden [6], $W$ can be written in terms of the principal stretches, namely

$$
W=W\left(\lambda_{1}-1, \lambda_{2}-1, \lambda_{3}-1\right)
$$

As with the invariant form, $W$ can either be a general function or an additive decomposition, that is

$$
W=\sum_{i=1}^{3} W_{i}\left(\lambda_{i}-1\right)
$$

For the incompressible case wherein

$$
\lambda_{1} \lambda_{2} \lambda_{3}=1
$$

the functional form of $W$ needs to be modified since any of the $\lambda_{i}$ can be replaced by a combination of the remaining measures, for example

$$
\lambda_{3}=1 /\left(\lambda_{2} \lambda_{3}\right)
$$

Based on the dependence depicted by the incompressibility, Equation (32), the invariants reduce to the form

$$
\begin{gathered}
I_{1}=\lambda_{1}^{2}+\lambda_{2}^{2}+\frac{1}{\left(\lambda_{1} \lambda_{2}\right)^{2}} \\
I_{2}=\left(\lambda_{1} \lambda_{2}\right)^{2}+\frac{1}{\lambda_{1}^{2}} \frac{1}{\lambda_{2}^{2}}
\end{gathered}
$$

$$
I_{3} \quad 1
$$

In view of Equations (32)-(35) and Equation (28), Equations (29) and (30) take the simpler forms

$$
\begin{gathered}
W=\left(I_{1}-3\right)+W_{2}\left(I_{2}-3\right) \\
W=W\left(\lambda_{1}=1, \lambda_{2}-1\right)
\end{gathered}
$$

or

$$
W=W_{1}\left(\lambda_{1}-1\right)+W_{2}\left(\lambda_{2}-1\right)
$$

As noted earlier, this can be sorted out during the curve fitting phase.

\section{REVIEW OF AVAILABLE FITS}

A wide variety of fitting schemes can be employed to establish a whole field model of material behavior. This includes such procedures as: (i) direct polynomial fits [14]; (ii) least squares [14]; (iii) orthogonal polynomials [9] [14]; (iv) Chebyshev type min-max fits [14]; and (v) rational polynomial fits [15]. Each of these procedures have various advantages and disadvantages. For instance, for direct polynomial fits, the Taylor like power series forms an infinite basis space. To establish a fit to large sample point sets requires a higher order polynomial. As order increases, such fits become very stiff as evidenced by the many order of magnitude span between the coefficients of the lower and higher order terms. Such stiffness leads to significant nonsample point errors, and especially to unbounded behavior outside the span of fitting. Note, while power series form a complete basis space in the Euclidean domain, higher order terms are not really admissible physically. Beyond perhaps the third order power, additional powers induce a growing model stiffness which is typically troublesome.

For least square fits a variety of functions can be employed to affect the fit, i.e. polynomials, transcendentals, and so on. While the procedure keeps the average square error down [14], unfortunately, extreme errors are permitted at nonsampling points [14]. Outside the fitting range, unstable unbounded asymptotes are common as higher order terms dominate [14]. When polynomials are employed, stiffness problems often occur.

For Chebyshev type representations, the fit is characterized by: (i) minimized extremal errors - the min-max principle; (ii) somewhat higher local square errors allowed; (iii) equal-ripple representations; (iv) best orthogonal polynomial fit. 
Note, the equal ripple property tends to lead to noisy derivatives. This is especially true when a min-max scheme is employed on higher order power series, i.e. polynomial functions. For such systems the stiffness induces increasing numerical instabilities. Note, while the Chebyshev min-max scheme is superior to the least squares approach, the stiffness issue still remains a problem.

Lastly, rational polynomials [15] can be employed to generate a fit. Overall, such functions are well suited to represent transcendental like responses. Altogether, rational polynomials possess the following properties: (i) can handle asymptotics about either the abscissa or ordinate axes within sample point ranges; (ii) remain rational under translation and scale change; (iii) remain asymptotically bounded beyond the sample point interval; (iv) as order increases, such fits become stiff for the same reasons as the direct polynomial fit.

Since integer polynomials are most commonly used to affect fits [2,3], the stiffness issue noted earlier can cause numerically unstable models. The Ogden [6] approach somewhat bypasses the problem by allowing the series powers to be established by the empirical data. In this context, irrational powers are often the norm [7]. Noting the very useful work of Finney and Kumar [7], the least squares scheme can be used to construct the requisite approximation. Beyond the usual difficulties noted earlier about least square fits, the major problem with free fractional power fits is one of uniqueness and existence. For multi-term representations, intervals of existence must be set to establish the optimal fractional powers. As the number of terms becomes larger, it is difficult to pre-assign the correct intervals. Nonetheless, the fractional formulation provides the best fit thus far. This is clearly seen by the work of Finney and Kumar [7].

To summarize issues, the problem with typical curve fitting-modeling procedures lies in the use of basis space functions which in their higher order form fail to be representative of a system's physical response characteristics. In this context, attempts to improve model accuracy will always give rise to increasing stiffness and numerical instability. By introducing free irrational powers, the Ogden approach [6] points to the potentials of fractional polynomial formulations. This will be investigated in the following sections.

\section{DIOPHANTINIZED FRACTIONAL POLYNOMIAL FITS}

In each of the foregoing formulations, an integer or free fractional power series type basis space was employed. As noted, each of these have various shortcomings. To bypass these, the current paper will consider the use of diophantinized fractional polynomial sets. Overall, the creation of such sets involves several main steps, namely: (i) establishing boundary order of the fit; (ii) selecting the diophantine [16] approximation for the fractional representation; (iii) first pass through least square fit for equally spaced sample points; (iv) iteratively remezing [8] the fit with a least square update; (v) contingent on error tolerance, redefine diophantine approximation set with new sample point distribution taken as interpolations of last remezed set; and (vi) redo (iv) and (v) contingent on tolerance test.

The philosophy and algorithmic structure of the above procedure is described in the following subsections.

\section{Natural bounding order}

For a given empirically defined data set, there are naturally occurring bounds on the local slopes. Given stress-strain/stretch data, this implies that

$$
B_{\mathrm{L}} \leqslant \frac{\mathrm{d}}{\mathrm{d} x}(f) \leqslant B_{\mathrm{U}},
$$

where $\left(B_{\mathrm{L}}, B_{\mathrm{U}}\right)$ are lower and upper bounds. Such bounds can easily be mapped out for the interval of interest. Note, the slope itself varies from point to point. The functional nature of this variation can be used to define the extremal orders of the diophantinized fit.

For a given local position, the dominant polynomial order induces the following functional proportionality namely

$$
f(x) \propto(x)^{\alpha},
$$

where here an origin centered fit is considered. Given that the response is monotone increasing, i.e. for $\forall x<\hat{x}, f(x)<f(\hat{x})$, then any two subsequent ordinate points can be used to define $\alpha$. In particular, for the pairs $((\mathrm{d} / \mathrm{d} \lambda)(x), x) ; \quad((\mathrm{d} / \mathrm{d} \lambda)(x+\Delta x)$, $x+\Delta x)$, we see from Fig. 1 that

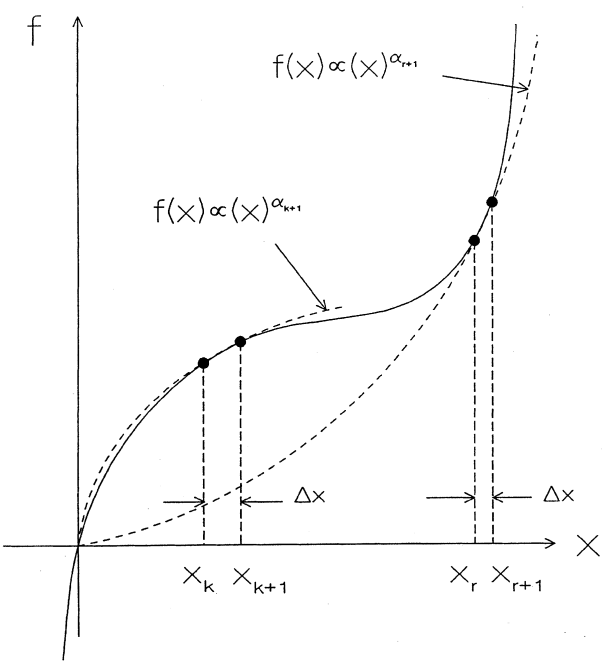

Fig. 1. Define pointwise origin centered order of empirical data set. 


$$
\begin{gathered}
\frac{\mathrm{d}}{\mathrm{d} x}(f(x)) \quad c \alpha(x)^{\alpha-1} \\
\frac{\mathrm{d}}{\mathrm{d} x}(f(x+x)) \quad c \alpha(x+x)^{\alpha-1}
\end{gathered}
$$

Solving Equation (41) and Equation (42) for $\alpha$, we obtain

$$
\alpha \quad 1+\frac{\log _{10}\left(f^{\prime}(x+x)-\log _{10}\left(f^{\prime}(x)\right)\right.}{\log _{10}(x+x)-\log _{10}(x)}
$$

Depending on the size of $x$, we can scan through the abscissa interval of interest to yield all ranges of $\alpha$ values, i.e.

$$
\alpha \quad 1+\frac{\log _{10}\left(f^{\prime}\left(x_{i}\right)-\log _{10}\left(f^{\prime}\left(x_{i-1}\right)\right)\right.}{\log _{10}\left(x_{i}\right)-\log _{10}\left(x_{i-1}\right)},
$$

where

$$
x_{i}=i \quad x ; i \in\left[0, I_{\mathrm{s}}\right],
$$

such that $I_{\mathrm{s}}+1$ is the number of sample points. The extremal values are then defined by the expressions

$$
\begin{gathered}
\alpha_{\min }=\min \left(\alpha_{i} ; i=1,2, \ldots, I_{\mathrm{s}}\right) \\
\alpha_{\max }=\max \left(\alpha_{i} ; i=1,2, \ldots, I_{\mathrm{s}}\right)
\end{gathered}
$$

The $\left(\alpha_{\min } ; \alpha_{\max }\right)$ extremal values bound the fit. In this context

$$
\begin{gathered}
f(\lambda) \quad \sum_{l}^{3} C_{1 l}\left(x_{l}\right)^{\alpha_{\min }}+\sum_{i=1}^{I_{\mathrm{f}}} C_{i+1 l}\left(x_{l}\right) \hat{\alpha}_{i} \\
+C_{I_{\mathrm{f}}+2 l}\left(x_{l}\right)^{\alpha_{\max }},
\end{gathered}
$$

where here $\left\{\alpha_{\min }, \hat{\alpha}_{i} ; i \in\left[1, I_{\mathrm{f}}\right] \alpha_{\max }\right\}$ defines a fractionalized basis space, and $I_{\mathrm{f}}+2$ denotes the number of fractional polynomial terms. The choice of $\hat{\alpha}_{i}$ will be discussed in the next section.

\section{Diophantine approximation}

The selection of $\hat{\alpha}_{i}$ can be achieved by a variety of procedures. For instance, the $\left\{\alpha_{i} ; i \in\left[1, I_{\mathrm{s}}\right], \alpha_{\max }\right\}$ family described by Equation (44) and Equation (45) can be employed to yield interpolated values of the $\left\{\alpha_{i} ; i \in\left[1, I_{\mathrm{f}}\right], \alpha_{\max }\right\}$ set. The interpolation can be taken either as: (i) equal spacings along the total sample point interval, Fig. 2; or (ii) equal spacings along the $\left(\alpha_{\min }, \alpha_{\max }\right)$ interval, Fig. 3 . In both cases completely irrational sets are obtained.

Alternatively, $\alpha_{\max }$ and $\alpha_{\min }$ can respectively be rounded up or down to the neighboring integer, i.e.

$$
\begin{aligned}
& I_{\mathrm{U}}=I\left(\alpha_{\max }\right) \\
& I_{\mathrm{L}}=I\left(\alpha_{\min }\right),
\end{aligned}
$$

where $I_{\mathrm{L}}<\alpha_{\min }$ and $\alpha_{\max }<I_{\mathrm{U}}$. The interval can then be disected to yield a diophantine type fractional family, namely

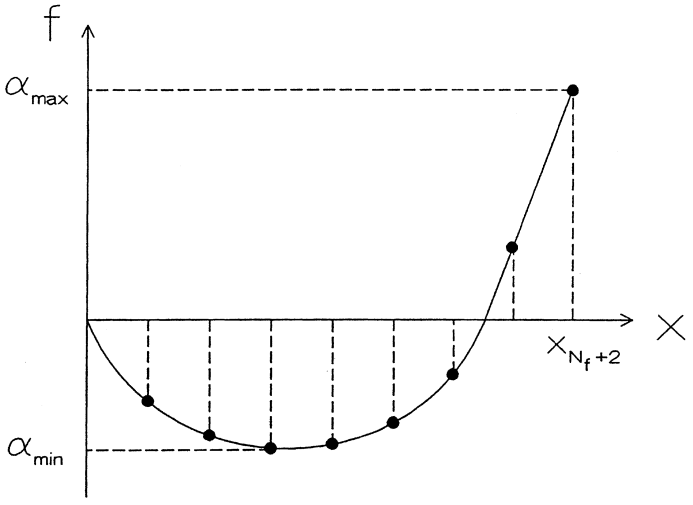

Fig. 2. Selection of $\hat{\alpha}_{i}$ set via equal spacings along sample point interval.

$$
\hat{\alpha}_{i}=I_{\mathrm{L}}+\frac{i}{N_{\mathrm{f}}+1}\left(I_{\mathrm{U}}-I_{\mathrm{L}}\right), i \in\left[1, N_{\mathrm{f}}+1\right]
$$

In this context, Equation (47) reduces to the diophantine form

$f(x) \quad \sum_{l} C_{1 l}\left(x_{l}\right)^{I_{\mathrm{L}}}+\sum_{i=1}^{I_{\mathrm{f}}} C_{i+1 l}\left(x_{l}\right)^{\alpha_{i}}+C_{I_{\mathrm{f}}+2 l}\left(x_{l}\right) I_{\mathrm{U}}$

\section{Least square}

Once order and the $\hat{\alpha}_{i}$ family has been established, the first iteration through the least square scheme involves equally spaced sample points. Two approaches are possible, namely: (i) the $\hat{\alpha}_{i}$ family is held fixed and an optimal solution is sought solely for the fitting coefficients $\underline{C}$; or (ii) the initial set $\left(\hat{\alpha}_{\min } ; \hat{\alpha}_{i} ; \hat{\alpha}_{\max }\right)$ is treated as seeding points for an iterative optimal search process enabling the solution of both the $\underline{C}$ and $\underline{\alpha}$ sets.

For the current purposes, since the $\alpha$ set can be continuously disected in the interval $\left(\hat{\alpha}_{\min }, \hat{\alpha}_{\max }\right)$, a

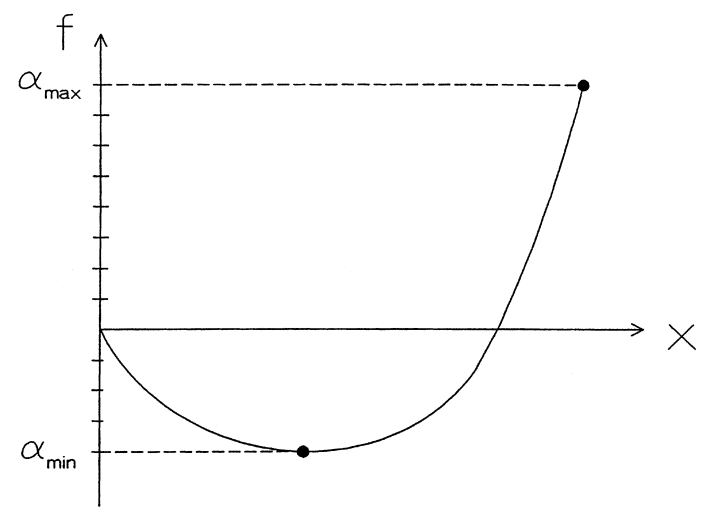

Fig. 3. Selection of $\hat{\alpha}_{i}$ set via equal spacings along the $\left(\alpha_{\min }, \alpha_{\max }\right)$ interval. 
complete fractional basis space can be formed. In this context, approach (i) is adopted herein.

\section{Remezing phase}

The main purpose of the remezing [8] phase is to seek an optimal spacing for the sample points. In this way the least squares scheme can yield an improved optimality. The remezing process consists of several operations. The central feature of the scheme involves the equalization of error throughout the interval of fitting. This is achieved by rearranging the sampling points in direct proportion to the level of error distribution. In this way, much like the Chebyshev type fit [14], remezing provides an equi-ripple min-max representation.

Algorithmically, the following steps are involved namely: (i) set number of integration points to define fitting error, $N_{\mathrm{I}}$; (ii) establish cubic spline fitted version of empirical data to represent "exact" behavior, $f_{\mathrm{e}}$; (iii) based on "exact" curve and least squares whole interval representation, form local error $f$ at each integration point $x_{i}$

$$
\begin{gathered}
x_{i}=i \frac{x_{\text {interval }}}{N_{\mathrm{I}}}=i x \\
f\left(x_{i}\right)=f_{\mathrm{e}}\left(x_{i}\right)-f\left(x_{i}\right) ;
\end{gathered}
$$

(iv) numerically integrate error over sample point interval

$$
E\left(x_{i}\right) \quad \sum_{l=1}^{i} 05 x\left(f\left(x_{l}\right)+f\left(x_{l}-1\right)\right),
$$

where here $i \in\left[0, N_{\mathrm{I}}\right] ;(\mathrm{v})$ given that there are $N_{\mathrm{f}}+2$ sample points, divide the net error into equal increments and determine distribution of such increments

$$
\begin{gathered}
E=\frac{1}{N_{\mathrm{f}}+2} E\left(N_{\mathrm{I}} x\right) \\
x \text { (samplepoints) } \equiv\left(x_{1}, x_{2}, \ldots, x_{N_{\mathrm{I}}}\right),
\end{gathered}
$$

where $x_{i}$ satisfy the condition

$$
E=\int_{x_{i-1}}^{x_{i}}\left\{f_{\mathrm{e}}(x)-f(x)\right\} \mathrm{d} x
$$

for $\forall i \in\left[1,{ }_{\mathrm{N}} \mathrm{f}+2\right]$, Fig. 4 .

\section{Iterative closure}

To round out the overall flow of calculations, the least square and Remez schemes are iteratively cycled through to optimally update: (i) fitting coefficients, $\underline{C}$; (ii) sample point distribution, $\underline{x}$; and (iii) diophantinized fractional powers, $\underline{\hat{\alpha}}$.

Figure 5 illustrates the flow of control. In the inner loop, the error check consists of comparing successive iterations of least square optimization and remezing. For the outer loop, $N_{\mathrm{f}}$ is updated to yield a densified diophantinized set of polynomial

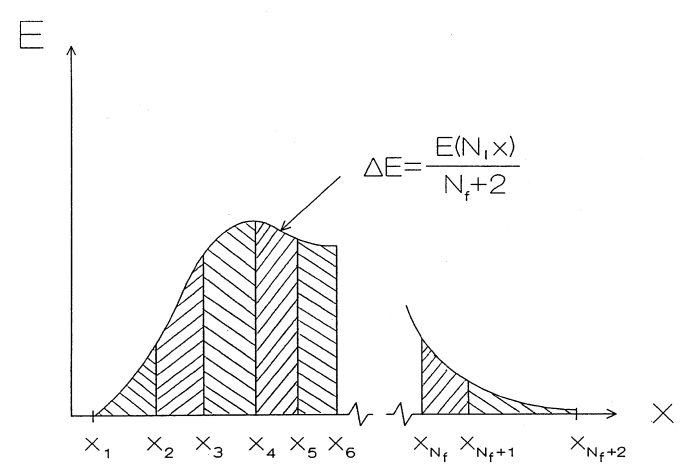

Fig. 4. Redefinition of sample point distribution by error equalization.

powers $\hat{\alpha}$. In the outer loop, as $N_{\mathrm{f}}$ is increased, the fractional set becomes more complete. Hence, the natural form of iterative cut off is meeting the predetermined tolerance requirement.

\section{FRACTIONAL POWER FITS FOR WORK-ENERGY AND STRESS VS STRETCH RATIO}

In this section, the diophantanized fractional polynomial fits will be specialized to develop workenergy/stress vs stretch ratio relations. For the current purposes, this will be undertaken for incompressible media. Two approaches will be considered, i.e. fractional polynomials in terms of either the

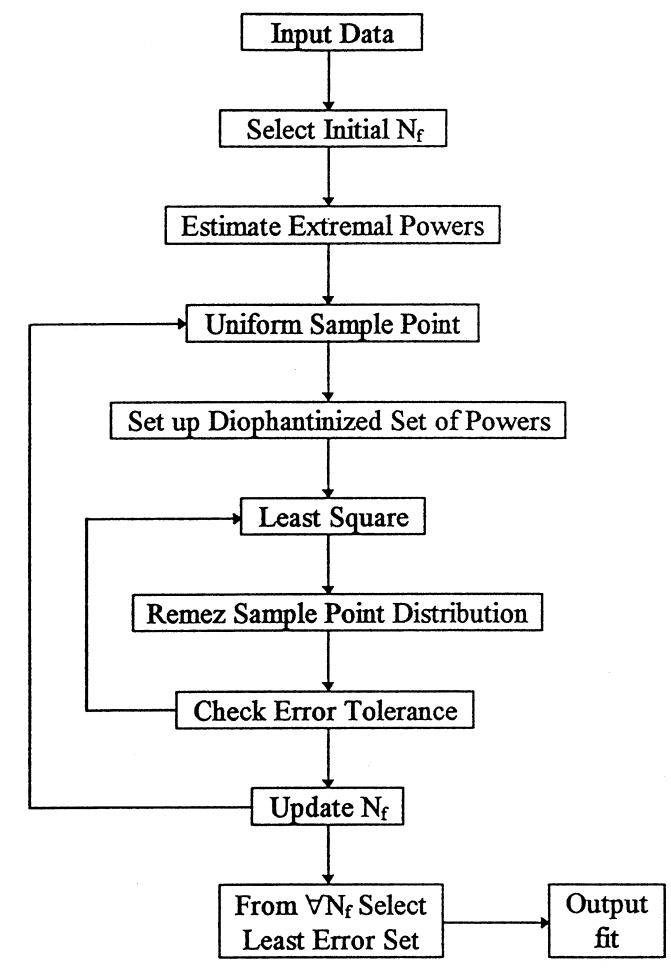

Fig. 5. Overall flow of calculations. 
principal stretch ratio or the strain invariants. These are described in the following subsections.

\section{Principal stretch model}

Recalling Equation (30), given isotropic media, to maintain directional independence it follows that

$$
W_{1}=W_{2}=W_{3}
$$

In this context expanding $W_{i}$ in fractional power series we obtain the relation

$$
W=\sum_{l=1}^{N_{\mathrm{f}}+2} \frac{C_{l}}{\alpha_{l}}\left\{\left(\lambda_{1}\right)^{\alpha_{l}}-1+\left(\lambda_{2}\right)^{\alpha_{l}}-1+\left(\lambda_{3}\right)^{\alpha_{l}}-1\right\}
$$

To determine the $C_{i}$ family, a variety of empirical tests can be employed, i.e. compression [1], tension [1], or shear. For such experiments, relation Equation (58) needs to be modified.

Consider the tensile and compression tests. In such situations

$$
\lambda_{2}=\lambda_{3}
$$

and through Equation (31) it follows that Equation (58) reduces to the form

$$
W=\sum_{l=1}^{N_{\mathrm{f}}+2} \frac{C_{l}}{\alpha_{l}}\left\{\left(\lambda_{1}\right)^{\alpha_{l}}+2\left(\lambda_{1}\right)^{-\alpha_{l} / 2}-3\right\}
$$

Based on Equation (22), Equation (60) yields the following expression of stress vs stretch ratio, that is

$$
1=\sum_{l=1}^{N_{\mathrm{f}}+2} C_{l}\left\{\left(\lambda_{1}\right)^{\alpha_{l}-1}-\left(\lambda_{1}\right)^{-\left(\alpha_{l} / 2\right)-1}\right\}
$$

In diophantinized form we get

$$
\begin{aligned}
1= & C_{l}\left\{\left(\lambda_{1}\right)^{\alpha_{\min }-1}-\left(\lambda_{1}\right)^{-\left(\alpha_{\min } / 2\right)-1}\right\} \\
& +\sum_{l=1}^{N_{\mathrm{f}}+2} C_{l}\left\{\left(\lambda_{1}\right)^{\alpha_{l}-1}\left(\lambda_{1}\right)^{-\left(\alpha_{l} / 2\right)-1}\right\} \\
& +C_{N_{\mathrm{f}}+2}\left\{\left(\lambda_{1}\right)^{\alpha_{\max }-1}-\left(\lambda_{1}\right)^{-\left(\alpha_{\max } / 2\right)-1}\right\},
\end{aligned}
$$

where here we must establish the bounding $\underline{\alpha}$ values; $\left(\alpha_{\min }, \alpha_{\max }\right)$.

Elastomers have a unique feature that when highly stretched, the slope in the stress-stretch space stiffens [1]. This, of course, is a result of molecular behavior as the long chains uncoil/untwist. We can take advantage of this property to determine a bound for $\alpha$. Specifically, in terms of the

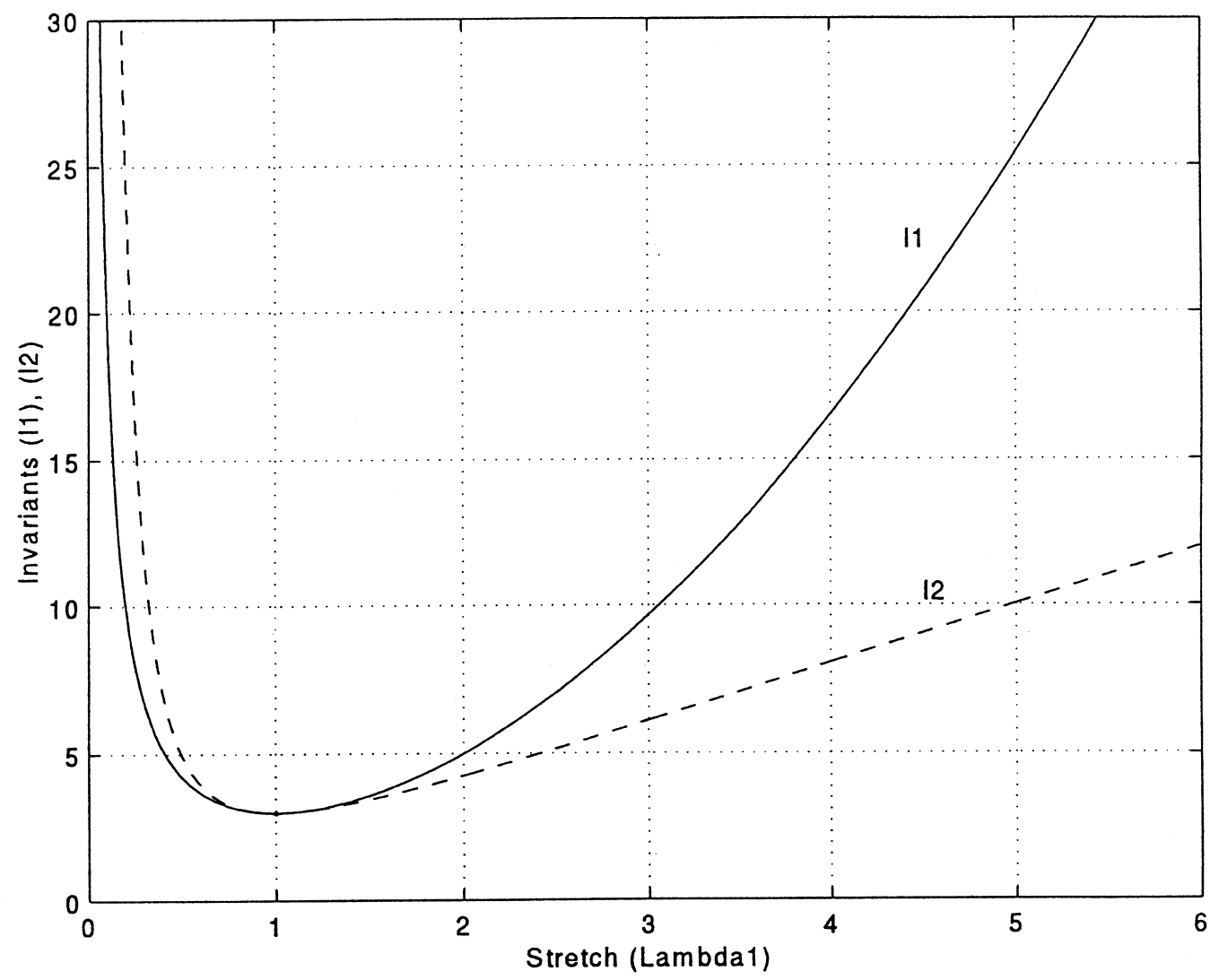

Fig. 6. Asymptotic trends of the invariants $I_{1}$ and $I_{2}$. 
discussion in Section 4, we need to evaluate either the function or slope at two adjacent $\lambda$ values. Given Equation (62), since the $C_{N_{\mathrm{f}}+2}$ term dominates for large $\lambda$,

$$
\left.{ }_{1}\left(\lambda_{1}\right) \propto C_{N_{\mathrm{f}}+2}\left(\lambda_{1}\right)^{\alpha_{\max }^{t}-1}-\left(\lambda_{1}\right)^{-05 \alpha_{\max }^{t}-1}\right],
$$

where $\alpha_{\max }^{t}$ is an upper bound for the tensile case. To determine the power, we ratio Equation (63) for subsequent $\lambda$. This yields the expression

$$
\frac{1\left(\lambda_{1 l}\right)}{1\left(\lambda_{1 l+1}\right)} \propto \frac{\lambda}{1 l}_{\lambda_{1 l+1}}^{\alpha_{\max }^{t}-1}\left\{\frac{1-\left(\lambda_{1 l}\right)^{-15 \alpha_{\max }^{t}}}{1-\left(\lambda_{1 l+1}\right)^{-15 \alpha_{\max }^{t}}}\right\},
$$

where here $\lambda_{1 l}<\lambda_{1 l+1}$ for $l \in\left[1, N_{\mathrm{f}}+2\right]$. For monotone increasing graphs,

$$
{ }_{1}\left(\lambda_{1 l}\right) /{ }_{1}\left(\lambda_{1 l+1}\right)<1
$$

and

$$
\left\{\frac{1-\left(\lambda_{1 l}\right)^{-15 \alpha_{\max }^{t}}}{1-\left(\lambda_{1 l+1}\right)^{-15 \alpha_{\max }^{t}}}\right]<1
$$

Solving Equation (63) for $\alpha_{\max }^{t}$ yields

$$
\alpha_{\max }^{t}=1+\frac{\log _{10}\left\{\frac{\frac{1}{1}\left(\lambda_{1 l+1}\right)}{1\left(\lambda_{1 l}\right)}\left(\frac{1-\left(\lambda_{1 l}\right)^{-15 \alpha_{\max }^{t}}}{1-\left(\lambda_{1 l+1}\right)^{-15 \alpha_{\max }^{t}}}\right\}\right.}{\log _{10}\left(\lambda_{1 l+1}\right)-\log _{10}\left(\lambda_{1 l}\right)}
$$

In view of Equation (66), it follows that

$$
\alpha_{\max }^{t} 1+\frac{\log _{10}\left({ }_{1}\left(\lambda_{1 l+1}\right)\right)-\log _{10}\left({ }_{1}\left(\lambda_{1 l}\right)\right)}{\log _{10}\left(\lambda_{1 l+1}\right)-\log _{10}\left(\lambda_{1 l}\right)}
$$

for large $\lambda_{1}$, i.e. $\lambda_{1} » 1$. Given the monotonicity of elastomer behavior, Equation (68) can be evaluated for the tail end of the response curve.

Under high compression, i.e. $\lambda<1$, polymers tend to also display significant stiffening. In such situations, Equation (63) asymptotically reduces to the form

$$
{ }_{1}\left(\lambda_{1}\right)-C_{N_{\mathrm{f}}+2}\left(\lambda_{1}\right)^{-05 \alpha_{\max }^{c}-1}
$$

Ratioing Equation (69) for subsequent monotonically decreasing $\lambda<1$ yields

$$
\frac{{ }_{1}\left(\lambda_{1 l}\right)}{{ }_{1}\left(\lambda_{1 l+1}\right)} \propto{\frac{\lambda_{1 l}}{\lambda_{1 l+1}}}^{-05 \alpha_{\max }^{c}-1}
$$

Based on Equation (70), we obtain the following expression for $\alpha_{\max }^{c}$, namely

$$
\alpha_{\max }^{c}=2 \frac{\log _{10}(1 l+1 / 1 l)}{\log _{10}\left(\lambda_{1 l} / \lambda_{1 l+1}\right)}-2
$$

As with Equation (68), Equation (71) can be estimated for the compressive tail end of the response curve. Note contingent on empirical data, either

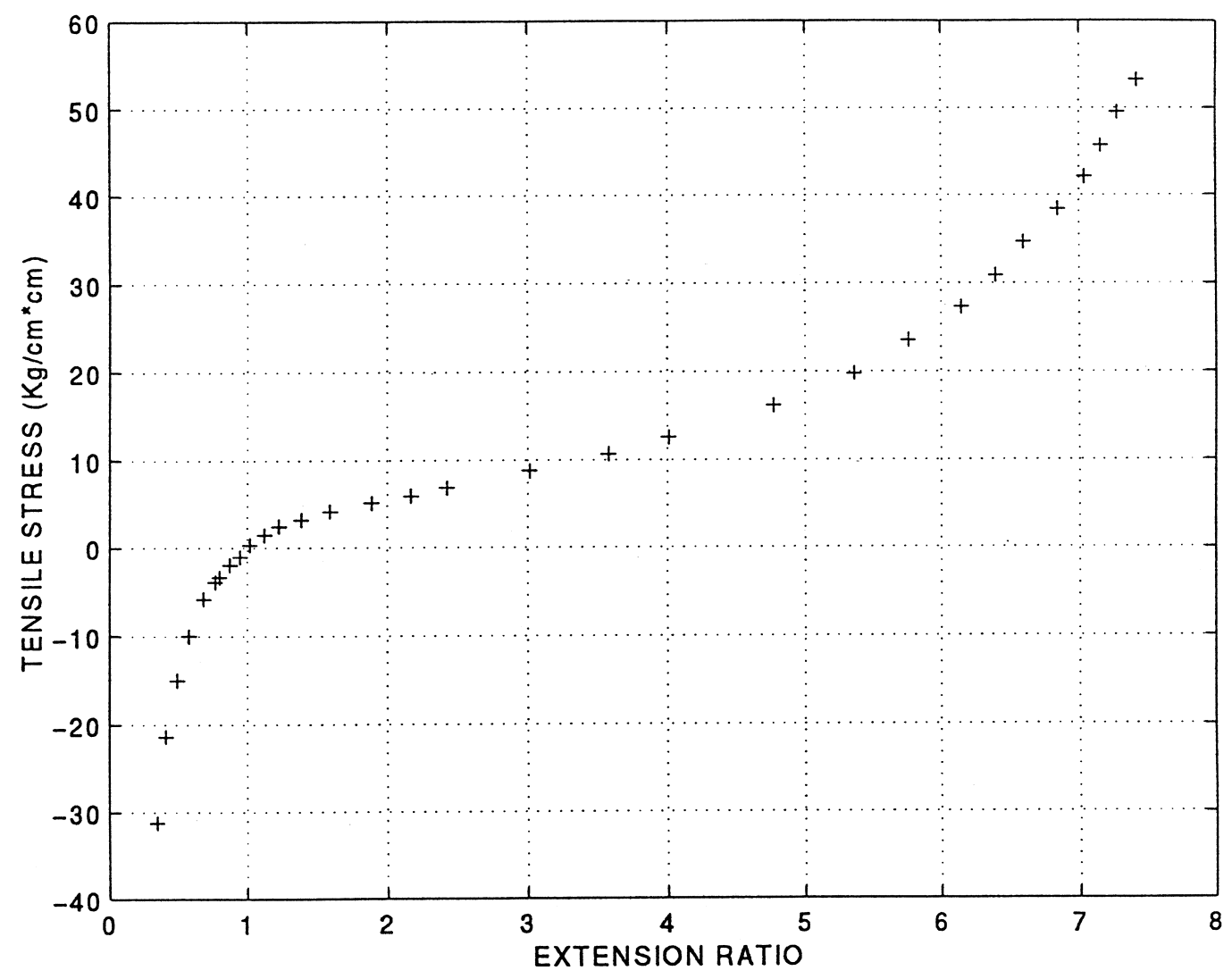

Fig. 7. Treloar rubber compression tension response data [1]. 
Equation (68) or Equation (71) can be used to set the greatest upper bound on polynomial power.

For minimum values of $\alpha$, Equation (64) needs to be solved iteratively for successive values of $\lambda$. Recasting the said equation in fixed point [14] algorithmic form, we yield the relation

$$
\alpha_{l+1}^{k+1}=\alpha_{\max }^{t}-\log _{10}\left(\xi_{l+1}\right) / \log _{10}\left(\lambda_{1 l+1} / \lambda_{1 l}\right),
$$

where

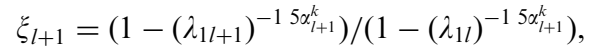

such that $k$ defines the iteration count. Note for $\lambda>1, \xi_{l+1}>1$ thus $\alpha_{\max }>\alpha$, as expected. Equation Equation (73) can be swept through the $\lambda$ range to establish $\alpha_{\min }$. For typical elastomers, this will occur for $\lambda \in$ (1, upper range).

Having established the extremum values of $\underline{\alpha}$, the full set can be constructed in the manner discussed. In this context, round $\alpha_{\max }$ and $\alpha_{\min }$ down to their nearest whole upper and lower bounding integers. Next, bisecting the integerized version of the noted interval yields the diophantinized set, i.e. Equation (49).

\section{Invariant model}

Before establishing the invariant form of the work-energy expression, it is useful to establish the asymptotic characteristics of $I_{1}$ and $I_{2}$. For incompressible media, it follows that

$$
\begin{gathered}
I_{1}=\lambda_{1}^{2}+\frac{2}{\lambda_{1}} \\
I_{2}=2 \lambda_{1}+\frac{1}{\lambda_{1}^{2}}
\end{gathered}
$$

Noting Fig. 6, for $\lambda_{1} » 1, I_{1}$ dominates, while for $\lambda_{1}<1, I_{2}$ is dominant. In this context, the bases of powers need to account for the $I_{1}$ and $I_{2}$ dependencies on $\lambda_{1}$. This leads to the following work-energy vs invariant formulation, namely

$$
W=\sum_{l=1} \sum_{i=1}^{2}\left(C_{l}^{i} / \alpha_{l}^{i}\right)\left(I_{i}-3\right)^{\alpha_{l}^{i}}
$$

Based on Equation (22) and Equation (76),

$$
\begin{aligned}
1 & =\sum_{l=1}\left\{C_{l}^{1}\left(I_{1}-3\right)^{\alpha_{l}^{1}-1} \frac{\partial}{\partial \lambda_{1}}\left(I_{1}\right)\right. \\
& \left.+C_{l}^{2}\left(I_{2}-3\right)^{\alpha_{l}^{2}-1} \frac{\partial}{\partial \lambda_{1}}\left(I_{2}\right)\right\}
\end{aligned}
$$

To bracket the $\underline{\alpha}_{l}^{1}$ and $\underline{\alpha}_{l}^{2}$ sets, we employ the asymptotics of $I_{1}, I_{2}$. Hence, for $\lambda \gg 1$, we see from Equation (74) and Equation (75) that

$$
1 \propto\left(I_{1}-3\right)^{\alpha_{l}^{1}-1} \frac{\partial}{\partial \lambda_{1}}\left(I_{1}\right)
$$

Ratioing Equation (78) for subsequent $\lambda_{1}$ values, on the upper bound tail end of the empirical data set, yields the constraint

$$
\alpha_{\max }^{1}=1+\frac{\log _{10}\left({ }_{1 l+1} I_{1 l, 1}\right)-\log _{10}\left({ }_{1 l} I_{1 l+1,1}\right)}{\log _{10}\left(I_{1 l+1}-3\right)-\log \left(I_{1 l}-3\right)},
$$

where $I_{1 l, 1}=\left(\partial / \partial \lambda_{1}\right)\left(I_{1 l}\right)$ and $I_{1 l+1,1}=\partial / \partial \lambda_{1}\left(I_{11+1}\right)$. For $\lambda_{1}<1$, we obtain the expression

$$
1 \propto\left(I_{2}-3\right)^{\alpha_{l}^{2}-1} \frac{\partial}{\partial \lambda_{1}}\left(I_{2}\right)
$$

Considering subsequent $\lambda_{1}$ values on the lower bound tail end, it follows that

$$
\alpha_{\max }^{2}=1+\frac{\log _{10}\left(1 l+1 I_{2 l, 1}\right)-\log _{10}\left({ }_{1 l} I_{2 l+1,1}\right)}{\log _{10}\left(I_{2 l+1}-3\right)-\log _{10}\left(I_{2 l}-3\right)}
$$

Equation (79) and Equation (81) can be evaluated

\begin{tabular}{|c|c|c|c|}
\hline$\lambda_{1}$ & 1 & $I_{1}$ & $I_{2}$ \\
\hline 0.350 & -31.20 & 0.84 & 0.86 \\
\hline 0.412 & 21.35 & 0.02 & 0.72 \\
\hline 0.493 & 15.00 & 0.30 & 0.11 \\
\hline 0.580 & 10.00 & 0.78 & 0.13 \\
\hline 0.685 & 5.80 & 0.39 & 0.50 \\
\hline 0.770 & 3.90 & 0.19 & 0.22 \\
\hline 0.800 & 3.40 & 0.14 & 0.16 \\
\hline 0.875 & 2.00 & 0.05 & 0.06 \\
\hline 0.950 & 1.05 & 0.01 & 0.01 \\
\hline 1.020 & 0.33 & 0.00 & 0.00 \\
\hline 1.125 & 0.47 & 0.04 & 0.04 \\
\hline 1.230 & 0.44 & 0.14 & 0.12 \\
\hline 1.390 & 0.22 & 0.37 & 0.30 \\
\hline 1.595 & 0.15 & 0.80 & 0.58 \\
\hline 1.890 & 0.13 & 0.63 & 0.06 \\
\hline 2.170 & 0.94 & 0.63 & 0.55 \\
\hline 2.425 & 0.83 & 0.71 & 0.02 \\
\hline 3.016 & 0.75 & 0.76 & 0.14 \\
\hline 3.580 & 0.66 & 3.38 & 0.24 \\
\hline 4.010 & 2.50 & 6.58 & 0.08 \\
\hline 4.770 & 6.13 & 3.17 & 0.58 \\
\hline 5.360 & 9.77 & 9.10 & 0.75 \\
\hline 5.760 & 3.51 & 3.52 & 1.55 \\
\hline 6.150 & 7.32 & 8.15 & 2.33 \\
\hline 6.400 & 0.89 & 1.27 & 2.82 \\
\hline 6.600 & 4.67 & 3.86 & 3.22 \\
\hline 6.850 & 8.40 & 7.21 & 3.72 \\
\hline 7.040 & 2.10 & 9.85 & 4.10 \\
\hline 7.160 & 5.73 & 1.54 & 4.34 \\
\hline 7.280 & 9.55 & 3.27 & 4.58 \\
\hline 7.420 & 3.23 & 5.33 & 4.86 \\
\hline
\end{tabular}
throughout the $\lambda_{1}$ interval to establish variations in the powers of $\left(I_{1}, I_{2}\right)$ terms. This can define bounding ranges, i.e.

$$
\begin{aligned}
& \alpha_{l}^{1} \in\left[\alpha_{\min }^{1}, \alpha_{\max }^{1}\right] \\
& \alpha_{l}^{2} \in\left[\alpha_{\min }^{2}, \alpha_{\max }^{2}\right]
\end{aligned}
$$

Given that each of Equation (82) are upper/lower bound integerized and equally bisected into $N_{\mathrm{f}}+2$ sets, we obtain:

$$
\alpha_{l}^{1} \quad I_{\min }^{1}+\frac{l}{N_{\mathrm{f}}+1}\left(I_{\max }^{1}-I_{\min }^{1}\right)
$$

Table 1. Compressive/tensile stress-stretch data 


$$
\begin{gathered}
\alpha_{l}^{2} \quad I_{\min }^{2}+\frac{l}{N_{\mathrm{f}}+1}\left(I_{\max }^{2}-I_{\min }^{2}\right) \\
\text { for } l \in\left[1, N_{\mathrm{f}}+1\right],
\end{gathered}
$$

such that $\left(I_{\min }^{1}, I_{\min }^{2}\right)$ and $\left(I_{\max }^{1}, I_{\max }^{2}\right)$ denotes the lower and upper bounding integers relative to $\left(\alpha_{\min }^{1}, \alpha_{\min }^{2}\right)$ and $\left(\alpha_{\max }^{1}, \alpha_{\max }^{2}\right)$, respectively. Note, as $N_{\mathrm{f}}$ is increased, the completeness of the fractionalized basis set is assured. The main advantage of such a basis is that all member functions possess powers bracketed by the properties associated with the empirical curve. This significantly reduces the stiffness of the fit.

\section{FRACTIONALIZED RATIONAL FITS}

As an alternative to polynomial representations, the use of rational forms is considered next. While it is possible to form a direct fit in stress-strain space, it is difficult to integrate the resulting rational polynomial, so as to yield the concomitant energy expression. To bypass this condition, the workenergy itself is expressed as a fractional ration polynomial. Sample point data can then be established by integrating the stress-strain data into the energy-strain space via a stable algorithm, i.e. the Romberg scheme [14]. Fitting can then be achieved via a direct approach with a min-max, i.e. Remez [8] phase, to optimize integrated sample point spacing so as to lead to a uniformly accurate result. The algorithms associated with such steps are described below.

As a first action, the stress-stretch space needs to be integrated over the range of available empirical data. To affect the best fit a Romberg scheme [14] is employed such that cubic splines [17] are used to fill in data gaps. If the data has some statistical scatter, local least square fitting can be employed along with the spline[17]. This leads to

$$
W\left(\lambda_{1}\right)={ }_{0}^{\lambda_{1}} 1\left(\lambda_{1}\right) \mathrm{d} \lambda,
$$

where here $\lambda_{1} \in\left[\lambda_{1}^{-}, \lambda_{1}^{+}\right]$. Contingent on the order of the rational representation and remezing requirements, $\lambda_{1}$ may be ranged over various points in the noted interval.

Note, the rational form can either be in terms of stretches or the invariants, namely

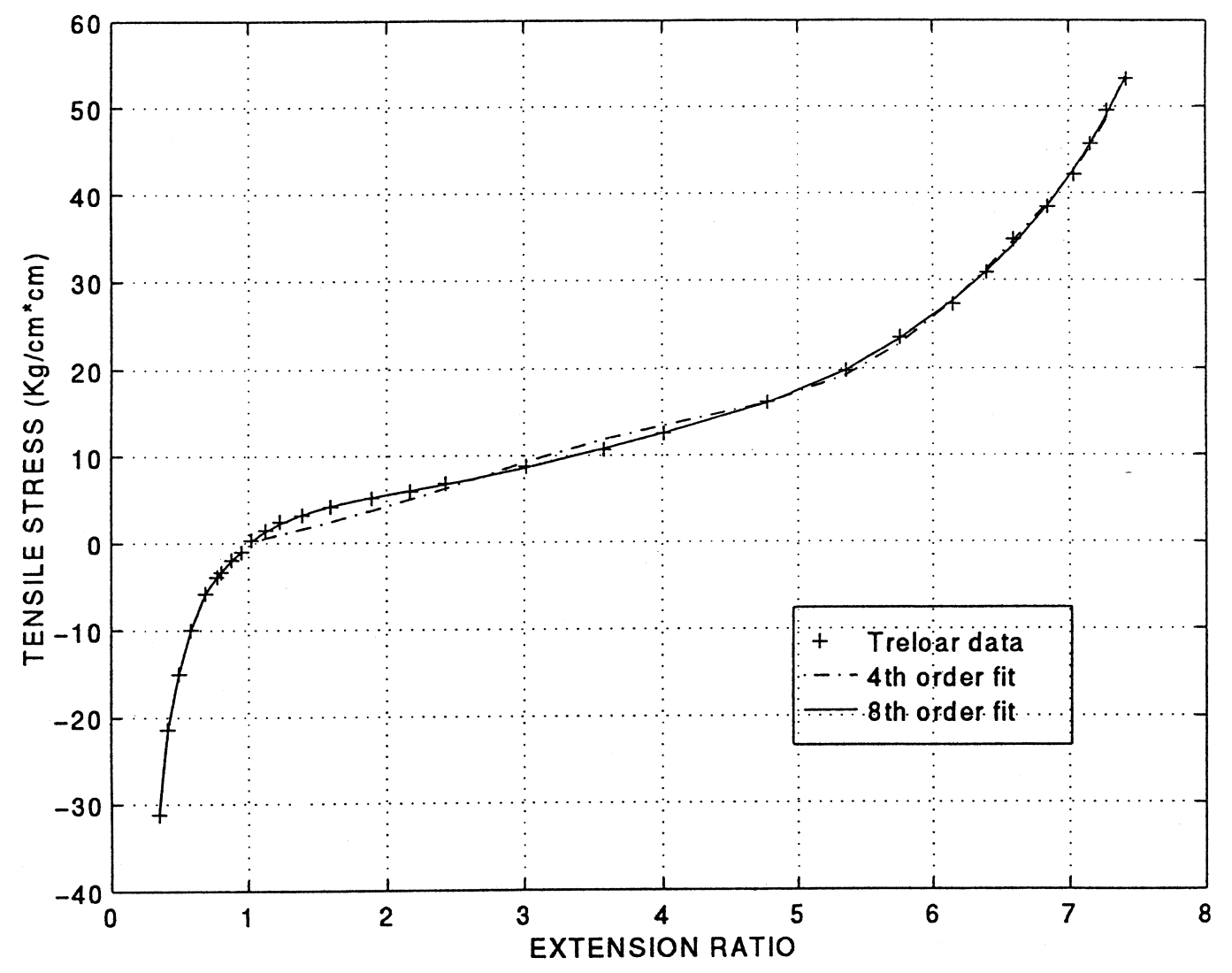

Fig. 8. Tension-compression fractional fit via principal stretch model-Treloar data. 


$$
\begin{array}{r}
\sum_{l=1}^{N_{\mathrm{f}}} \sum_{i=1}^{2} C_{l i}^{D}\left(I_{i}-3\right)^{l} \\
W=\frac{\sum_{l=1}^{N} \sum_{i=1}^{N_{\mathrm{f}}} \sum_{l i}^{2} C_{l i}^{N}\left(I_{i}-3\right)^{\alpha l}}{\sum_{l=1}^{N_{\mathrm{f}}+1} \sum_{i=1}^{2} C_{l i}^{D}\left(I_{i}-3\right)^{\alpha l}}
\end{array}
$$

or

$$
W=\frac{\sum_{l=1}^{N_{\mathrm{f}}} C_{l i}^{N}\left(\left(\lambda_{1}-1\right)^{\alpha_{l}}+\left(\lambda_{2}-1\right)^{\varepsilon_{l}}+\left(\lambda_{3}-1\right)^{\alpha_{l}}\right)}{\sum_{l=1}^{N_{\mathrm{f}}+1} C_{l i}^{D_{l i}}\left(\left(\lambda_{1}-1\right)^{\alpha_{l}}+\left(\lambda_{2}-1\right)^{\alpha_{l}}+\left(\lambda_{3}-1\right)^{\alpha_{l}}\right)}
$$

The denominators have a raised power to provide for stable asymptotics at the tails of the fit. For incompressible media, Equation (31) applies. Considering tension and compression empirical test, Equations (85)-(87) reduce to the form

$$
\begin{aligned}
& W= \\
& \frac{\sum_{l=1}^{N_{\mathrm{f}}} C_{l 1}^{N}\left(\lambda_{1}\right)^{2}+\frac{2}{\lambda_{1}}-3^{l}+C_{l 2}^{D} 2 \lambda_{1}+\frac{1}{\lambda_{1}^{2}}-3^{l}}{\sum_{l=1}^{N_{\mathrm{f}}+1} C_{l 1}^{D}\left(\lambda_{1}\right)^{2}+\frac{2}{\lambda_{1}}-3^{l}+C_{l 2}^{D} 2 \lambda_{1}+\frac{1}{\lambda_{1}^{2}}-3^{l}}
\end{aligned}
$$

Equations (86)-(88) can be solved through a combination of either direct or least square fitting with a remezing phase which optimizes the sample point placement. This, in effect, makes the results more uniformly accurate throughout the region of fitting.

\section{SAMPLE APPLICATIONS}

Because of the coiling/twisting and kinking/folding actions of elastomers, their compressive and tensile responses are quite distinct. This of course, leads to significant curve fitting difficulties. In many industrial applications, components most often involve compression, tension, or combination actions [18]. This often leads to significant modeling

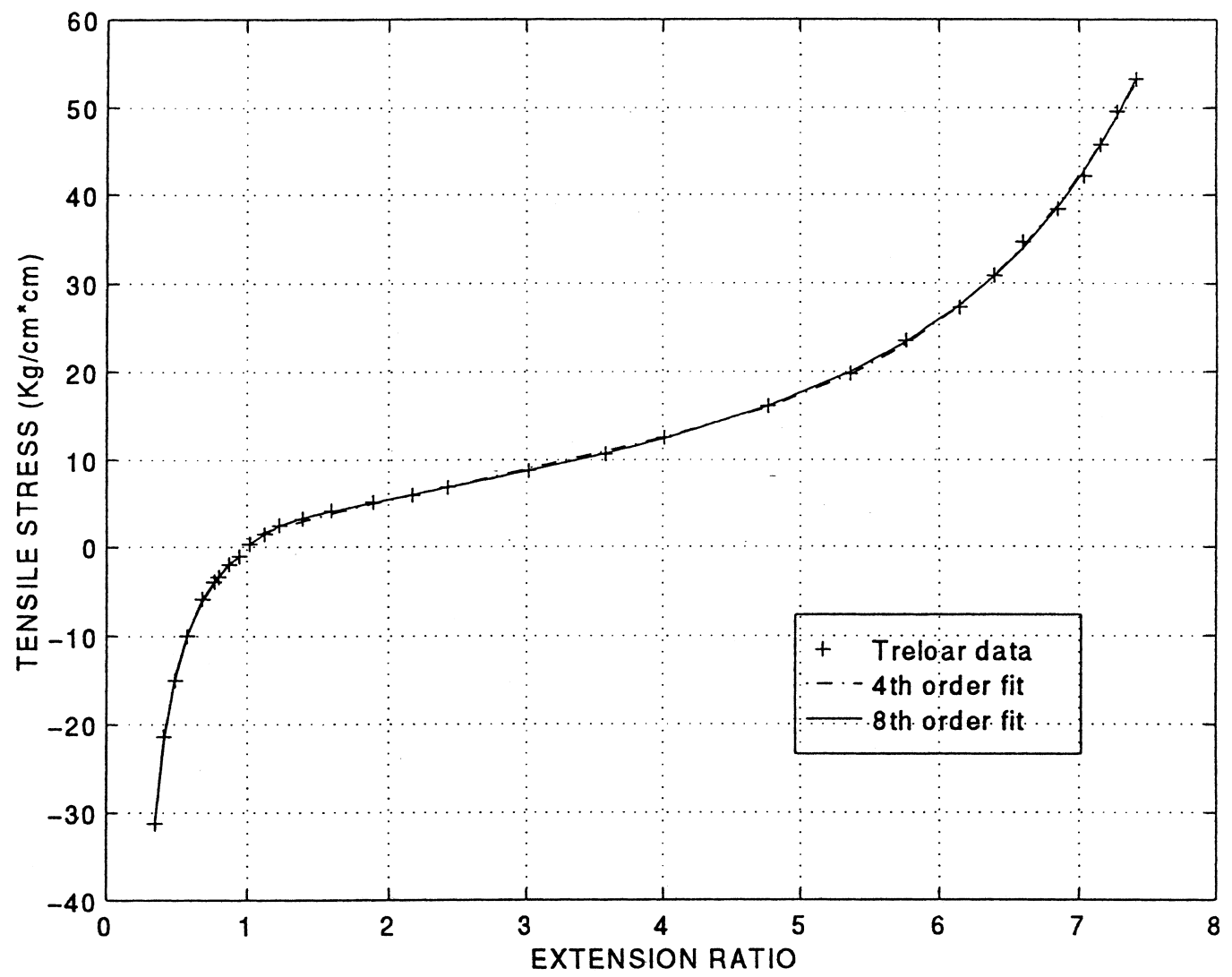

Fig. 9. Tension-compression fractional fit via invariant model-Treloar data. 
instability problems when improper stiffnesses are represented. Noting the compression-tension response reported by Treloar [1], Fig. 7, it follows that the compressive actions are significantly stiffer than the tensile. In this context, using tension only data to fit the response behavior will lead to either anomalous structural response behavior, local instabilities, folding, or to a variety of modeling problems: excessive element distortion, negative pivots, ill-conditioned Jacobians, or iterative convergence problems.

To quantify the numerical accuracy of the diophantinized polynomial and rational expressions, the empirical data of Treloar [1], Fig. 7, will be modeled. This will be done for both the invariant and stretch formulations. Recalling the previous sections, in the case of polynomial type fits, the flow of fitting calculation involves several steps, i.e. (i) defining diophantinized powers; (ii) performing iterative interactions between least square and remezing algorithms; and (iii) updating diophantinized set of powers to yield the required accuracy.

Based on the data given in Fig. 7 and Table 1, Figs 8-11 list the accuracy correlations associated with the fit types listed below: (i) stretch formulated-integer and fractionalized; (ii) invariant formulated - integer and fractionalized.
To gage the error, a Euclidean type norm is employed, namely

$$
E_{\epsilon}(\text { Euclidean error })=\frac{\sqrt{\sum_{l}\left(\left(\lambda_{l}\right)-{ }^{\mathrm{a}}\left(\lambda_{l}\right)\right)^{\overline{2}}}}{\sqrt{\sum_{l}\left(\left(\lambda_{l}\right)\right)^{\overline{2}}}},
$$

where ()$^{\mathrm{a}}$ defines the approximated data.

A main feature of the problem of fitting is the performance as one increases the order of the fit. Generally, a proper well-defined scheme will yield $E_{\epsilon}$ which diminishes monotonically as the order is raised. As can be seen from Fig. 10, for the integer scheme, the accuracy degenerates for large orders. In particular, beyond a problem dependent order, the integer fit whether involving stretches or invariants tends to become unstable numerically. The level of achievable accuracy is thus also problem dependent. In contrast, the fractional scheme yields monotonically improving results as the number of terms, i.e. base functions are increased. The sources of error for the integer scheme are several fold, namely: (i) the inadmissibility of the base functions leads to problem stiffness; (ii) the matrix formulation of the least square algorithm typically lacks diagonal dominance - this is particularly exacerbated by higher order integer fits; and (iii) for a

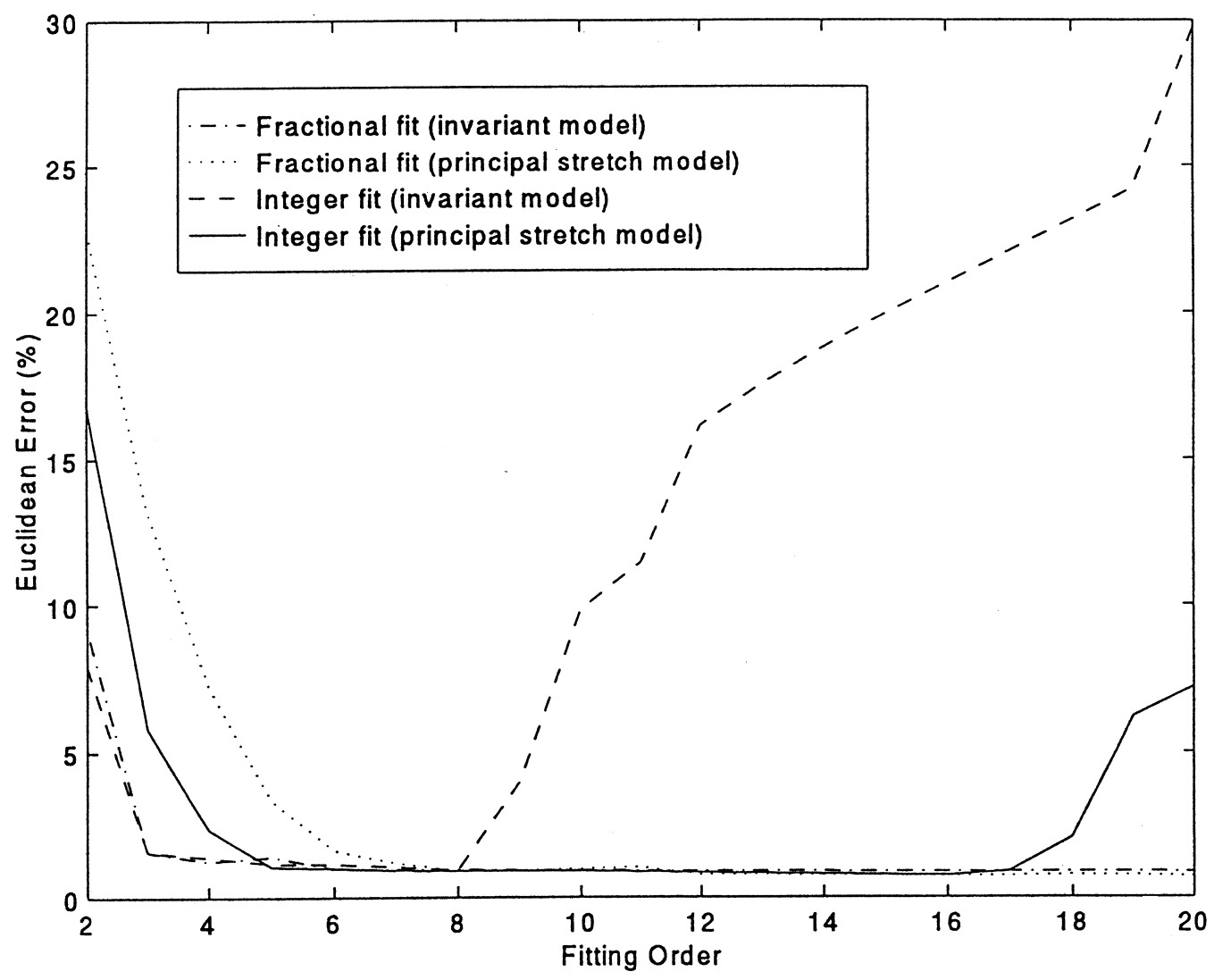

Fig. 10. Comparative Euclidean error behavior-tension range. 


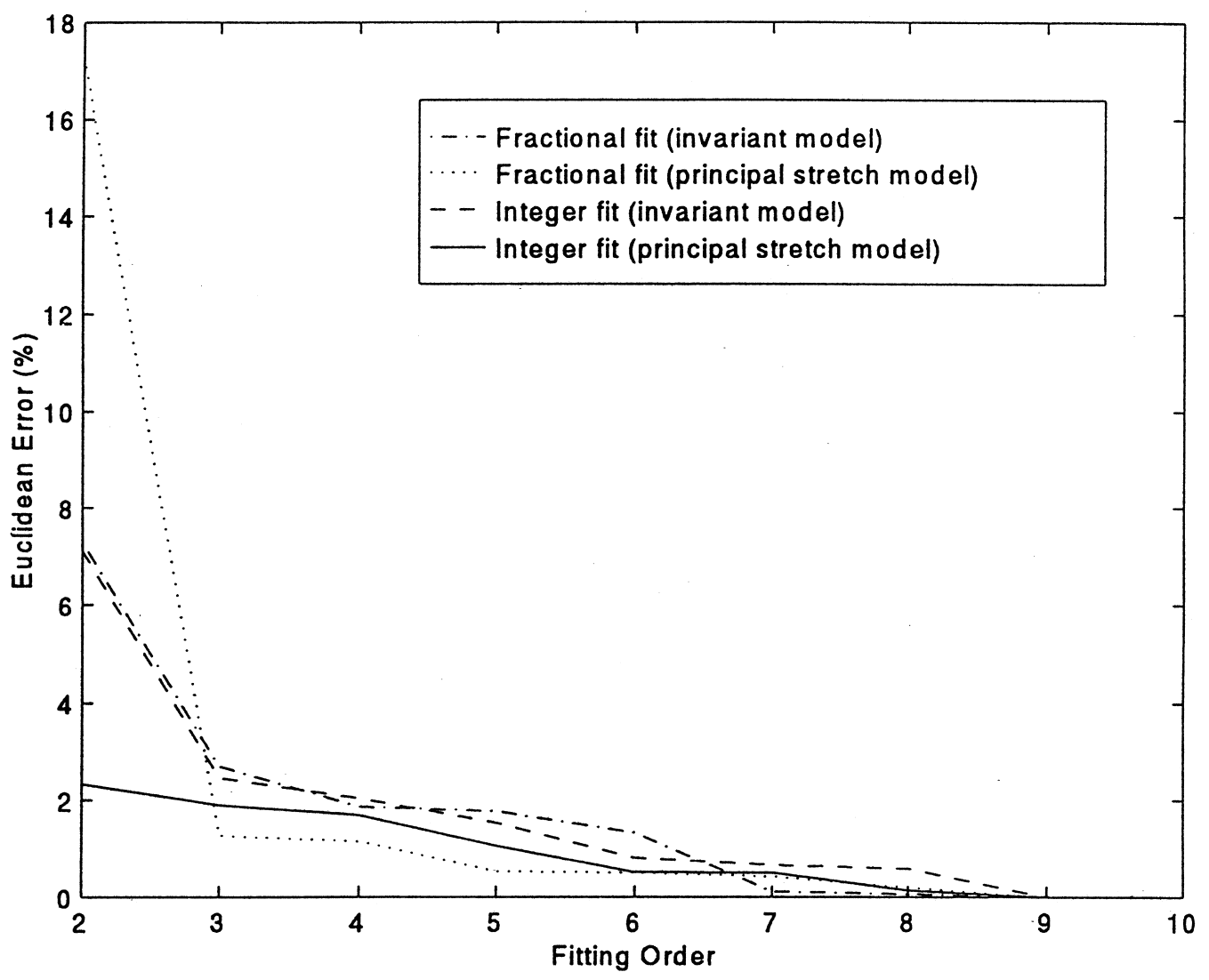

Fig. 11. Comparative Euclidean error behavior-compression range.

given computer accuracy, the problem stiffness and matrix ill-conditioning leads to significant round off, particularly as the number of base terms are increased.

The foregoing behavior implies that the integer scheme is highly sensitive to machine accuracy. For large scale applications, computer accuracy needs have a very strong influence on resource allocation requirements, i.e. memory, computational speed, data transfer rates, addressing schemes, etc. Generally computer architecture is optimized around a certain threshold accuracy, namely single, double, quadruple precision. For calculations which require increased accuracy, fairly substantial - super-linear increases in effort occur. This is due to increased addressing, bus traffic, and conversions of hardwired computational operations to less optimal formulations. This is clearly illustrated when one evaluates single and double precision results for a given LINPACK test-matrix inversion. Note, for the given calculations, the traditional double precision mode was employed (16 places).

The preceding monotonically improving rates of convergence apply for both the fractional stretch and invariant formulations, whether applied in a purely polynomial or rational form. For the Treloar data, the invariant approach showed a modestly improved fitting correlation. Which functional type - polynomial vs rational-is essentially a matter of preference. In conclusion, the diophantine approximated fractional form of constitutive relation yields an improved representation of three-dimensional material properties for elastomeric media. This applies for both the tensile and compressive ranges.

Acknowledgements - The partial support of the EPIC organization for the first author is gratefully acknowledged.

\section{REFERENCES}

1. Treloar, L. R. G. Transactions Faraday Society, 1944 , 40, 59 .

2. Mooney, M. Journal of Applied Physics, 1940, 11, 582.

3. Rivlin, R. S. Philosophical Transactins, Royal Society, London, 1948, 240A, 459.

4. Peng, S. T. J., Nonlinear multiaxial finite deformation investigation of solid propellants, AFRPL, TR-85-036, 1985.

5. Peng, S. T. J. and Landel, R. F. Journal of Applied Physics, 1975, 46, 2599.

6. Ogden, R. W. Proceedings of the Royal Society, London, Series 4, 1972, 328, 567.

7. Finney, R. H. and Kumar, A. Development of material constants for nonlinear finite-element analysis. Rubber Chemistry and Technology, 1988, 61, 879-891. 
8. Remez, E. Y. Sur le Calcul Effectiv des Polynomes d'Approximation de Tchebichef. Comptes Rendues de l'Academie des Sciences, Paris, 1934, 199, 337-340.

9. Meinardus, G., Approximation of Functions; Theory and Numerical Methods. Springer, New York, 1967.

10. Carpenter, A. J. and Varga, R. S., Some numerical results on best uniform polynomial approximation of $x^{\alpha}$ on $[0,1]$. In Methods of Approximation Theory in Complex Analysis and Mathedmatical Physics, eds Gonchar, A. A. and Saff, E. B. Springer, New York, 1991.

11. Farhad, T. Elastic stability of rubber products. Rubber Chemistry and Technology, 1987, 60, 957-965.

12. Malvern, L. E., Introduction to the Mechanics of a Continuous Medium. Prentice Hall, Englewood Cliffs, NJ, 1969.
13. Fung, Y. C., Foundations of Solid Mechanics. Prentice Hall, Englewood Cliffs, NJ, 1965.

14. Hamming, R. W., Numerical Methods for Scientists and Engineers. McGraw-Hill, New York, 1962.

15. Dold, A. and Eckmann, B., Rational Approximation and Interpolation. Springer, New York, 1983.

16. Edixhaven, B. and Evertse, J. H., Diophantine Approximation and Abelian Varieties. Springer, New York, 1993.

17. Bojanov, B. D., Hakopian, H. A. and Sahakian, A. A., Spline Functions and Multivariate Interpolations. Kluwer Academic, Boston, MA, 1993.

18. Padovan, J., Tabaddor, F., Gent, A. and Johnson, K. Surface wrinkles and local bifurcations in elastomeric components; seals and gaskets; parts I and II theory and application. Journal of Finite Elements in Analysis and Design, 1991, 9, 193-227. 